\title{
Effects of the World's Oceans on Global Climate Change
}

\author{
Vadim V. Navrotsky \\ Department of Physical Oceanology, V. I. Il'ichev Pacific Oceanological Institute, Far Eastern Branch, \\ Russian Academy of Sciences, Vladivostok, Russia \\ Email:vnavr@poi.dvo.ru
}

Received March 28, 2013; revised May 2, 2013; accepted May 27, 2013

Copyright (C) 2013 Vadim V. Navrotsky. This is an open access article distributed under the Creative Commons Attribution License, which permits unrestricted use, distribution, and reproduction in any medium, provided the original work is properly cited.

\begin{abstract}
The role of the World Ocean in Global Climate Change is considered from two points of view: 1) heat energy accumulation and distribution in the ocean and its discharge into the atmosphere as purely physical processes; 2) participation of living matter in the ocean in these processes. The oceanic organic matter, especially plankton and different organic compounds, absorbs solar energy and changes water transparency, controlling thickness of layers and amount of the energy accumulated. Having ability to react not only to fluctuations of solar heat energy supply, but also to extra weak fluctuations of electromagnetic and magnetic fields of terrestrial and extraterrestrial origin, phytoplankton and other organic matter should be considered as active forcing of global climate and ocean ecosystem fluctuations observed on different scales. Several mechanisms of solar activity effects on global climate-ocean ecosystem interactions are discussed.
\end{abstract}

Keywords: Global Climate; Ocean Ecosystems; Phytoplankton; Solar Activity; Ultraviolet Radiation; Magnetic Field

\section{Introduction}

Global Climate Change (GCC) and its consequences are among the crucial problems for contemporary civilization. We consider Global Climate (GC) as a spatialtemporal distribution over the Globe of an ensemble of hydrometeorological (climatic) parameters, such as temperature, air pressure, wind, cloudiness, humidity, precipitations, patterns of intra annual variations etc., averaged over some conditional spatial and temporal scales $L_{a}$ and $T_{a}$. Taking different values for $L_{a}$ and $T_{a}$, we will receive different levels of Global Climate specification. The climatic parameters values are defined by processes in the Earth Climate System (ECS) that includes oceans, atmosphere, land, biota and human civilization as a particular sub-system. Climate fluctuations are manifestation of energy fluctuations in the ECS, and the most important part of that energy is contained in space-time dispersions of climatic parameters.

In simplified global climate descriptions the main generally used parameter is temperature, which corresponds, to some extent, to energy content in the ECS. But it is not sufficient to adequately represent climate because it does not include heat energy transformations into kinetic and potential energy of atmosphere and oceans. Such transformations are determined by heat content gradients in oceans, atmosphere and especially between land and ocean. These gradients are due primarily to uneven income and uptake of solar energy over the globe and then to heat advection in oceans and atmosphere. They define energy of climate fluctuations, that is, spacetime dispersions of climatic parameters, which are for the all living creatures the really perceptible most important features of Global Climate.

The other problem, tightly connected to GCC, is the state of the Earth biota, which includes oceanic and land ecosystems. Though GC is formed by interaction of processes in the all parts of ECS, in most of actual investigations climate fluctuations are isolated and considered as drivers of fluctuations in the Earth ecosystems. Concerning oceans, characteristic examples are international conferences "Effects of Climate Change on the World's Oceans" in Gijon, Spain, 2008 and in Yeosu, Korea, 2012; "Ocean Climate and Marine Ecosystems", Busan, Korea, 2011, and hundreds of relevant papers. As a matter of fact, Global Climate and Earth ecosystems are unclosed highly nonlinear systems, which both are affected by processes from outside the ECS. Though there are many valuable results concerning GCC effects on physical and biological processes in the ocean, it seems reasonable from the cause-and-effect point of view to show usefulness and even necessity also of the inverse way of thinking and investigations: from ecosystems to Global 


\section{Climate Change.}

\section{Climate Change and the World Ocean: Energy Considerations}

It is doubtless that the main source of energy in ECS is solar radiation. To understand the role of oceans in climatic processes on purely physical basis several estimates for distribution of solar energy will be useful. It was shown in many studies summarized in [1], that energies of evaporation and heat advection in oceans are about two orders more than energy of atmosphere movements. At the same time, energies of fuel combustion and consumption by contemporary Mankind are comparable to the energy of atmosphere movements, and therefore, must have appreciable impact on climate. That means that relatively small change of energy of evaporation or ocean heat advection can lead to considerable relative increase of kinetic and potential energy and water content in the atmosphere and to the all processes, which we are observing as climate change.

In this connection very important are estimates in IPCC AR4 Synthesis Report, WG1, Ch. 5 [2]. Heat content change (in units of $10^{22} \mathrm{~J}$ ) in the period 1961-2003 was 14.2 in oceans, 0.5 in atmosphere and 0.76 on continents from global total 15.9. It means that oceans assimilated near $90 \%$ of global heat content change. It was about 30 times more than in atmosphere and about 20 times more than on continents. The analogous values for the period 1993-2003 were 8.11 in oceans, 0.20 in atmosphere and 0.18 on continents. Corresponding changes in heat content in oceans were 40 times more than in atmosphere and 45 times more than on continents. Relations of mean annual rates of heat accumulation in the periods 1993-2003 and 1961-2003 were 3 for oceans, 2.1 for atmosphere and 1.23 for continents. From these estimates two points are very important: 1) oceans are overheated relative to atmosphere and continents; 2) the overheating was going on with acceleration during the several last decades. There is not any doubt that the World Ocean is the main "storehouse" of solar heat energy, but so high and quick magnification of solar energy in oceans comparatively to land and atmosphere means that Global Warming begins from oceans, and it would be reasonable to investigate the impact of World's Oceans on Climate Change as the primary process.

Apart from energy for photosynthesis in plants, there is no considerable long-term heat energy accumulation on continents (though there is accumulation of cold in form of ice). In the ocean much more solar energy can be assimilated: 1) because albedo is lower, than on land; 2) because solar radiation can directly penetrate to depths of order $100-150 \mathrm{~m}$ and spread to depths of several hundred meters with the help of turbulence. Some part of that energy is reradiated back into the atmosphere, but the main part of it is accumulated in vertical and horizontal structure of ocean waters. Due to latitudinal and land-ocean contrasts of solar energy influx, that energy participates in heat advection by the ocean and atmosphere currents, increasing land-ocean temperature contrasts, forming energetically active zones and driving the basic circulation of the Earth atmosphere. Fluctuations of accumulated in the ocean energy are main drivers for Global Climate Change, and we should look for mechanisms of changes in the ocean assimilating and accumulating properties.

\section{Climate Change, Sun and Life in the Ocean}

It is evident that penetration and accumulation of solar energy in oceans depends on water clarity, and the main cause of change in clarity is change of organic matter concentration. Some part of solar light is used by phytoplankton (PP) for photosynthesis, but from the point of climate the main effect of plankton and accompanying organic matter is to shade subsurface waters from solar energy penetration. The depth of solar heat penetration is not strongly limited by optical properties of water. Due to multi-scale vertical and horizontal mixing, upwelling, downwelling and deep ocean circulation it can be accumulated at all depths and in all parts of the ocean. In that way large part of solar energy becomes "fossil" energy, which may not exhibit itself in climatic processes during long time. Heat content anomalies in layers deeper, than the main thermocline, can appear and participate in ocean-atmosphere interaction after they are transported with velocities of order $5 \mathrm{~cm} / \mathrm{s}$ to the upwelling regions, and that transport can take $10-20$ years or more. Effects of these "fossil" anomalies are interacting with effects of actually generated surface-layer anomalies, and the observed trends in climatic parameters can result from rather random coincidence of accumulation-discharge shifts in different oceans. In any case, due to the oceans, there is no possibility to obtain strictly zero balance for the Earth heat income-outcome on the decadal scale.

Using estimates of total primary production in the ocean about $50 \times 10^{9}$ tons of organic carbon and energy of $120 \mathrm{Kcal} / \mathrm{mole}$ expended for production of one mole of sugar (72 $\mathrm{g}$ of $\mathrm{C}$ in $180 \mathrm{~g}$ of sugar), we receive solar energy of order $0.5 \times 10^{21} \mathrm{~J} / \mathrm{yr}$ in oceans and total $1.0 \times$ $10^{21} \mathrm{~J} / \mathrm{yr}$ used for photosynthesis in plants on our planet. Large part of that energy is spent for oxygen production, and it is of the same order as energies of fuel combustion and consumption by Mankind. It means that we are burning almost all oxygen supplied by plants, correspondingly augmenting concentration of carbon dioxide.

There are obvious PP affecting processes, such as wind, temperature, nutrients supply etc., but they are local, defined by actual state of the Climate System, and 
hardly can lead to trends in Global Climate. We should look for external global drivers, and the main external global factor is solar emanation. Though phytoplankton is sensitive to water temperature, but the globally averaged change of temperature of order $1^{\circ} \mathrm{C}$ is much smaller, than its intra-annual fluctuations of order $5^{\circ} \mathrm{C}-20^{\circ} \mathrm{C}$ for any PP population, and it cannot globally influence PP biomass. So, it is natural to believe that PP and ocean water properties can change and have considerable anomalies not only due to climatic processes, but also due to the caused by solar activity specific changes in solar emanation properties.

Changes of solar activity are traditionally connected with sunspots, which are described by Wolf numbers Wn. To study the structure of $\mathrm{Wn}$ in detail, we used series of its daily values for the period 1818-2006 [3], and corresponding spectrum is shown in Figure 1.

The main peak corresponds to the generally recognized 11-year cycle. Less prominent cycle of 22 years is due to change of polarity in the 11-year cycles, the period of 5.7 years can be a harmonic of the main cycle, the other cycles can have different explanations. The prominent peak at 27 days corresponds to the period of solar rotation (in low latitudes), the small 13.5 and 7-day peaks can be due to sectorial structure of the solar magnetic field. Special attention should be given to the peaks in the range of 4 - 5-year and 2 - 2.5-year periods, which are found frequently in climatic parameters fluctuations, ENSO as an example.

Using the notion of instantaneous frequency and decomposition into intrinsic mode functions (basis), proposed in [4] for spectral analysis of nonlinear and nonstationary processes, the series of daily Wn fluctuations during 55 years was analyzed (Figure 2).

We can see that practically all energy of fluctuations is enclosed within well separated 11-year segments. Amplitudes of fluctuations between the segments are negli-

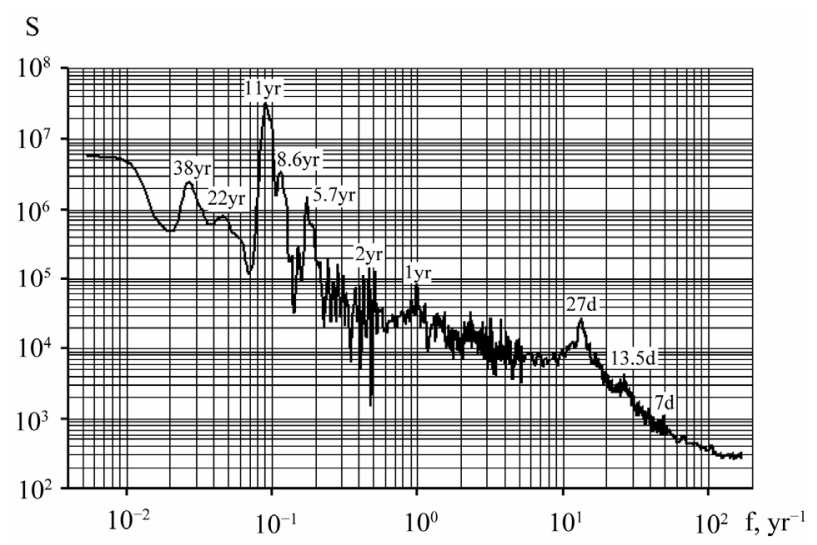

Figure 1. Spectrum of daily values fluctuations of Wn for the period 1818-2006 (in logarithmic scales). The values for frequencies higher than $10 \mathrm{yr}^{-1}$ are smoothed by 5 points.

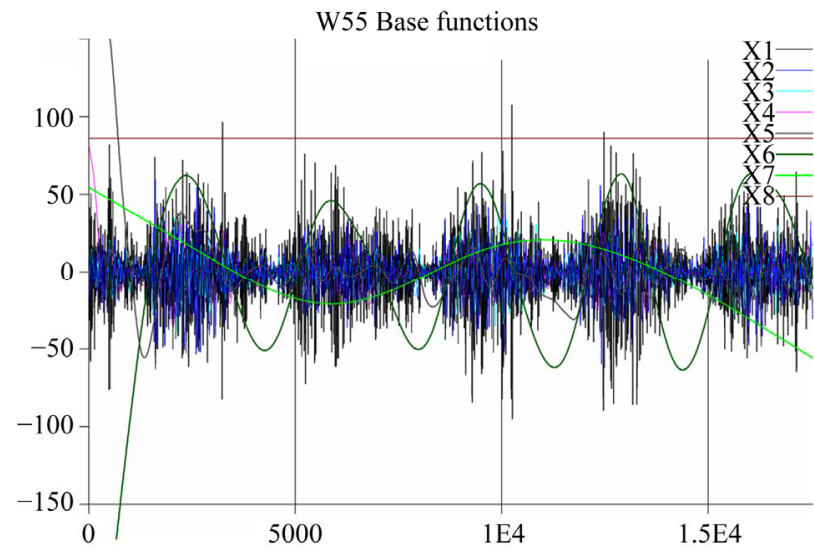

Figure 2. Intrinsic mode functions (basis) of daily wolf numbers fluctuations during 1950-2004.

gible. The 11-year component looks like an envelope of many shorter-period components, marking only beginning and end of the shorter-period disturbances. Detailed analysis of the basic functions in Figure $\mathbf{2}$ has shown that components 1 and 2, embracing periods from 5 till 45 days, can have amplitudes higher, than the component 6 , clearly representing 11-year cycle. Though in integral spectrum the short-period components are much lower than the 11-year maximum, they can be more stable from one cycle to another and so be well pronounced in the time-frequency spectrum of series including many cycles. To clarify the situation with sunspots, the time-period (or time-frequency) spectrum of $\mathrm{Wn}$ fluctuations during 1906-2006 was calculated (Figure 3).

Analysis of Figure 3 leads to interesting results: 1) Columns of maximum spectral energy grow out of the zone, corresponding to 11-year periods ( $T=$ about 4000 days). Relative long-term temporal changes of energy in the 11-year component are very small, while in shorterperiod components we see rather sharp alternation of high and very low levels of energy with time; 2) Spectral structure of fluctuations in time-spans comprising $\mathrm{Wn}$ maximum (energy distribution in columns) changes considerably from one cycle to another practically not repeating, though zones of energy maxima with periods 600 - 900 days and 60 - 40 days can be seen inside most cycles; 3) The most striking feature is strong regularity of energy changes in the range 5 - 40 days: in each cycle energy moves from initial periods to smaller ones and then returns to initial periods at the same energy level independently of considerable changes of spectral structure at longer periods. That process is very similar to behavior of classical inertial Kolmogorov's turbulence, and we can suppose that the shortest periodicity of $\mathrm{Wn}$ fluctuations (from days till 1 - 4 weeks) points to the range of viscose for velocity and diffusive for magnetic field energy dissipation in sunspots.

Apparently, there are large-scale processes with perio- 


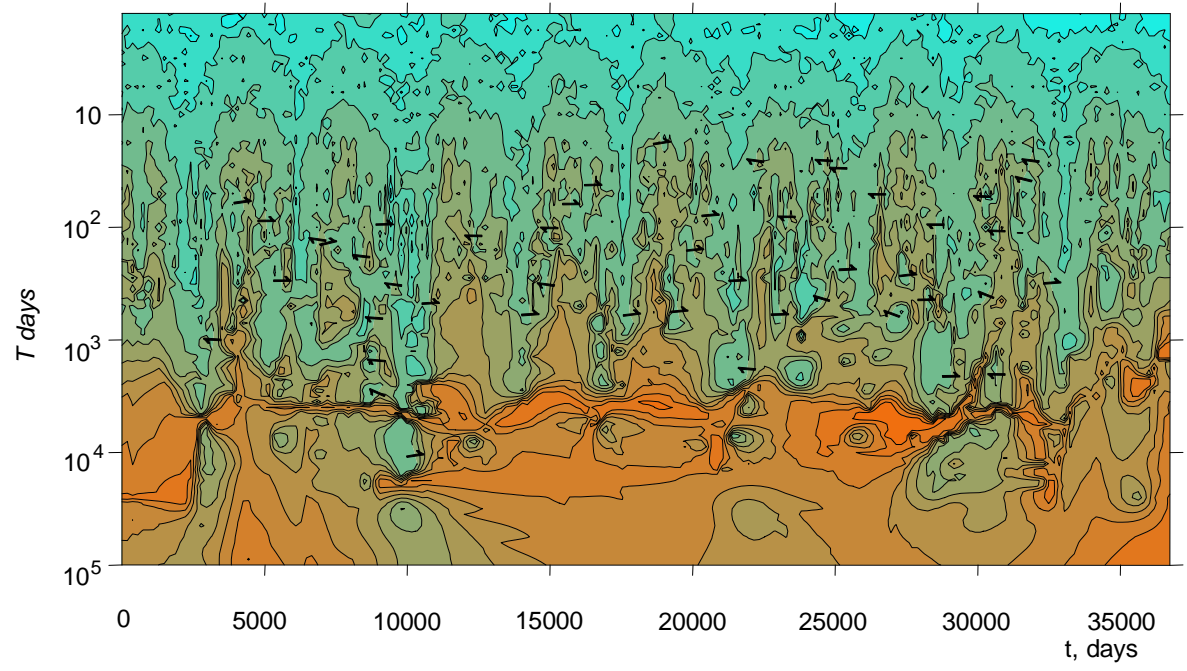

Figure 3. Hilbert-Huang spectrum of Wolf number fluctuations during 1906-2006. Periods $T$ and energy are shown in logarithmic scale.

dicity about 11 years, leading to change of polarity of the solar magnetic field. But physically we should study not only 11-year connections between solar and very different terrestrial processes, but also mechanisms of repeated manifold effects of many short-living processes, connected with sunspots - integral radiation, flares, corpuscular flows, EM frequency spectrum fluctuations, magnetic fields. It was shown that fluctuations of integral radiation of order $0.1 \%$ can not explain the observed considerable trends in global temperature [5], and their direct effect on global biota must be negligible as well.

Biological effectiveness of electromagnetic fields is dependent on the wave length, and it is especially high in the range of ultraviolet irradiance UV-B at wave lengths $280-315 \mathrm{~nm}$ [6-8]. Relative fluctuations of that irradiance caused by different solar events can be much higher than relative fluctuations of total solar radiation. Tight connection of UV radiation with sunspots can be seen in Figure 4, where 27-day smoothed daily fluctuations of UV-index taken from [9] and daily values of Wn [3] for the same period and in the same way smoothed, are shown. Practically linear relation between the processes allows estimating UV levels basing on much easier measured Wolf numbers Wn. Among different periods, characteristic for solar activity, UV has periodicity in the short-period range about 30 days (which includes inherent scales of phytoplankton and zooplankton reproduction) and quasi-biennial periodicity, reaching maximum in the years of east phase and minimum in the years of west phase of ENSO [9].

Effects of fluctuations in solar UV-B irradiance on Global Climate are complex. 1) The main effect of UV in the ocean is photoinhibition, when considerable reduction in the photosynthetic capacity of a plant takes place. High levels of UV during some periods will lead to de- crease of solar energy spent for new production and to corresponding increase of energy left for water heating. Effects of UV on DNA can lead to mutations and changes in structure of populations; 2) Decrease of PP concentration and biomass leads to better water transparency, that is, to deeper solar light penetration and accumulation-about $100 \mathrm{~m}$ in oligotrophic waters in comparison to 10 - $30 \mathrm{~m}$ in eutrophic waters; 3) UV breaks down molecules of dissolved organic carbon (DOC) and other organic matter, which strongly attenuates the light penetration and effectively absorbs in the UV range. The produced organic compounds are consumed by bacterioplankton. Water transparency increases, that is, UV cleanses the way for solar energy and UV deeper penetration.

The above named processes define general tendencies, but they are realized very non-uniformly in space and time depending on many non-global factors. First, the effects will be best pronounced in highly productive regions, which in many cases coincide with energetically active zones of the World Ocean. Second, with similar PP concentration, the effects will be maximum in zone with maximum solar illumination, that is, in tropics. Third, effects of UV irradiation can considerably differ in coastal and open ocean due to difference in prevailing PP species (Li et al., 2011). The most important consequences of PP concentration and corresponding light penetration fluctuations for ocean and atmosphere circulation are analyzed by Gnanadesikan and Anderson [10] using a coupled climate model.

Very interesting consequences follow from the fact that different species of PP have differential sensitivity, that is, different ability to stand against UV-induced damages [11]. That means that during the periods of enhanced UV radiation some changes in structure of plank- 

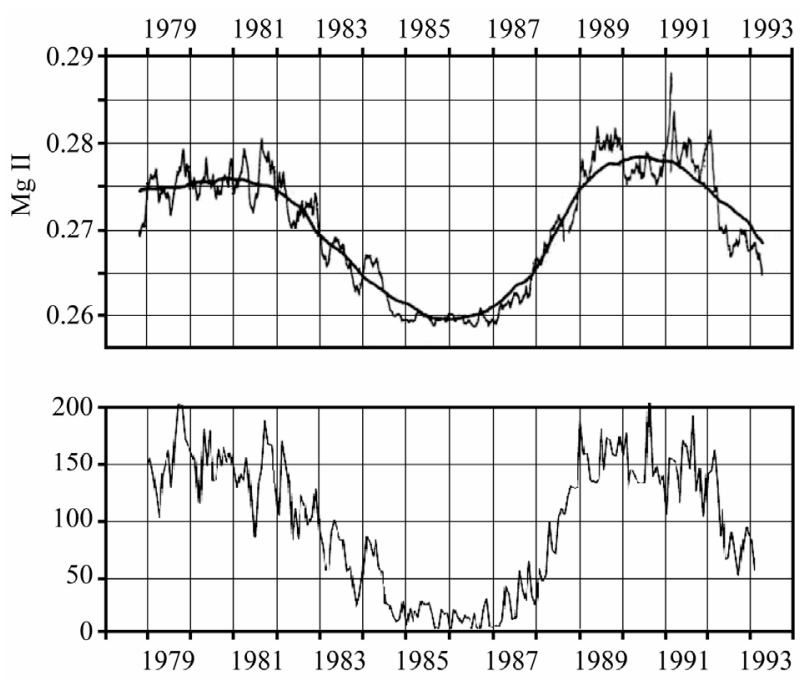

Figure 4. Variations of UV index Mg II [9] and Wolf numbers Wn.

ton communities take place. Less damaged species get preferences among competitive species and produce exceptionally high biomass, diminishing water transparency. Solar heat concentrates in thin surface layers, and extremely heated surface water extremely heats adjacent atmosphere. In that way can arise water temperature and atmosphere pressure anomalies characteristic for El-Niňo, which can be not so the cause, as a result of the specific large-scale blooms caused by solar activity fluctuations.

Effects of solar activity are not limited to ultra violet radiation. Less studied, but may be not less important are fluctuations of magnetic/electromagnetic (EM) fields. That phenomenon is based on the fact that very high internal field in bio membranes leads to strong polarization of biological objects and possibility of resonances between intrinsic elastic oscillations in the membranes and magnetic/electromagnetic oscillations. It was shown that nonlinear coupling of elastic and electric polar modes and additional energy supply can create a quasi-ferroelectric behavior in cells. Coherent oscillations will lead to collective biochemical reactions through non-linear long-range interactions, and consequently, to extraordinary high sensitivity of biological systems to extremely weak magnetic/electromagnetic signals [12]. So, we should take into account non-thermal bioeffects of solar radiation: processes with energy much lower, than needed to produce thermal effects (such processes can be treated as informational), can trigger macroscopic processes in living systems, affecting their activity, metabolism, and productivity.

The impacts from different parts of solar radiation spectrum occur, though differently, in all parts of trophic chains. It means that changes in ecosystems and in their influence on climate are due not only by bottom-up, but top-down forcing as well. As a result, the information, received by biological systems, controls macroscopic processes in the environment and in that way contributes into Global Climate and Global Ecosystem Changes.

Some kind of informational abilities have also lyotropic solutions, because they can change their structure without changing chemical composition and thermodynamic state. Biological liquids are just lyotropic solutions with high sensitivity to extra weak fluctuations of EM and magnetic field, and they can form an additional way of non-climate effects on living matter and subsequent impact of the oceanic living matter on Global Climate. Magnetic field fluctuations due to connected with flares coronal mass injections can affect not only living matter, but ocean water as well. There is not enough information about effects of magnetically changed water on metabolism of different organisms and species in oceans, but already known experimental results (changes of surface tension, viscosity, size of water clusters, proportion of free molecules etc.) clearly point to the necessity of studies in that direction $[13,14]$.

\section{Discussion}

Investigations of solar influences on the Earth climate are conducted in many directions, and many different mechanisms were proposed to relate these influences with solar activity. The main attention was given to the 11year and 22-year cycles. Concerning sun-climate-ocean relations, the most substantial and interesting results were obtained by $\mathrm{W}$. White with colleagues $[15,16]$. It was shown that the largest amplitudes of energetic response to the sun induced fluctuations are in tropical regions of the oceans, in mid-latitudes, and in sub-polar regions. Situation with tropics is natural, because there is maximum of available solar irradiance, but for the two other regions we suggest maximum of ocean-atmosphere interactions and maximum plankton concentration fluctuations as additional explanations.

Heat content anomalies caused by externally forced changes of PP in mid-latitudes concentration will lead to fluctuations of atmosphere circulation and corresponding fluctuations of currents velocities. That signal, positive or negative, will propagate up-stream in boundary currents like Golf Stream or Kuroshio and lead to velocity fluctuations in equatorial currents. Low velocity of equatorial currents leads to higher heat accumulation and vice versa. At the same time, if we estimate differences of heat content in the layers $0-20 \mathrm{~m}$ and $0-100 \mathrm{~m}$ with the same solar radiation, but different PP concentration, we come to the conclusion that anomalies in plankton biomass, caused by fluctuations in Sun emanation spectral structure, even in tropics can have greater effect on heat content, than fluctuations of general irradiation. Some support to that statement can be obtained from the result of Tourre and White [15] that anomalies of surface tem- 
perature and heat content in layers $0-400 \mathrm{~m}$ are opposite in phase (the less organics - the greater heat content of the layer, but less sea surface temperature, and vice versa). In any case, the both mechanisms, physical and biological, do not contradict, but supplement each other.

White et al. [16] have shown that depth-weighted vertical average temperature (from the sea surface till the top of the main pycnocline) are significantly correlated with changing surface solar radiative forcing in Indian, Pacific, Atlantic, and Global Oceans with lags $0 \pm 2$ years. But the negative lag at -2 years needs explanation beyond the direct forcing by change in surface solar radiation and green-house effects. Such explanation can be obtained, if we take into account that mechanisms of anomalies in PP and other organics concentration are not necessarily in phase with visible solar irradiation, and the discussed effects of UV for heat content can appear earlier, than via mechanism of total solar radiative forcing and large-scale ocean-atmosphere interaction.

It was shown in $[17,18]$ that observed Quasi-Decadal Oscillations in climate and ecosystems are well described by mechanism of delayed action oscillations (DAO), and they are due to damped resonance between 11-year cycles of solar activity and thermal processes in oceans. The result depends, to some extent, on addition of 11year-period cosine signal of amplitude $\sim 2.0 \mathrm{~W} \cdot \mathrm{m}^{-2}$ to the solar constant. The discussed effects of PP may amplify effects of solar constant fluctuations or be in phase with internal physical processes in some regions and considerably help in explaining and predicting anomalies of thermal, dynamic and biological processes on regional and basin scales. Theoretically, in nonlinear systems, resonances between intrinsic high-frequency fluctuations in small parts of a system and external forcing with additional energy supply will lead to low-frequency largescale fluctuations through non-linear long-range interactions. So not only 11-year, but much longer cycles can result from the solar forcing.

Active role of ocean living matter in Global Climate implies that changes of ecosystem characteristics can forestall climate changes with different lags. Examples of such possibilities can be seen in [19], where relationships of Pacific salmon and Japanese sardine catches with Atmospheric Circulation Index (ACI) in the Northern Pacific are shown for the periods 1920-1950 and 1970-1993. Among others, there are presented four pictures for unsmoothed series where the catches maxima are 1 - 3 years ahead of maxima in climate parameters.

Analogous interdependence can exist between trends in mean sea surface temperature and areas of oligotrophic waters in Atlantic and Pacific oceans. In [20] and several earlier papers expansion of oligotrophic waters in subtropical gyres is explained by surface layer warming leading to thermocline intensification and corresponding decrease in fluxes of nutrients. Not discussing here some contradictions and doubts about that hypothesis (intensification of atmosphere circulation with global warming and corresponding deepening of upper thermocline as an example), it is worth to note that the discussed phenomena can be explained by the reverse processes: reduction in PP and other organics abundance under influence UV radiation leads to increment of solar energy in upper layers and corresponding increase in temperature. Taking into account that heat content in oligotrophic waters is extremely sensitive to PP concentration (which substantially depends on UV fluctuations) and that such waters occupy the largest part of pelagic oceans, they can be responsible for considerable input in global climate fluctuations.

To show clearly the needed ways of observational and modeling studies corresponding to the idea about simultaneous direct action of solar and geophysical factors on biological and climatic processes and their subsequent interaction, the simplified scheme is constructed including also anthropogenic impacts (Figure 5). Solid lines show direct impacts, arrows indicate their directions. Dashed lines with arrows in both directions indicate mutual feedbacks between subsystems. Lines 1, 2, 3, 4 show impacts of solar emanation. Lines 5, 6, 7 show natural interactions in the Earth Climate, lines 8, 9, 10 show the role of mankind in the Earth System.

The scheme assumes possibility of box modeling, but including of solar forcing independently on the all subsystems makes the problem much more difficult, than in existing coupled models of ocean-atmosphere interactions and models of climate impacts on regional or basin-scale ecosystems. First, solar impacts along lines 1, 2, 3 , and 4 in many cases can be different in their physical mechanisms, amplitudes, periodicity, and relevance for the subsystems. Second, all subsystems have their internal laws of evolution and eigenvalues of inherent fluc-

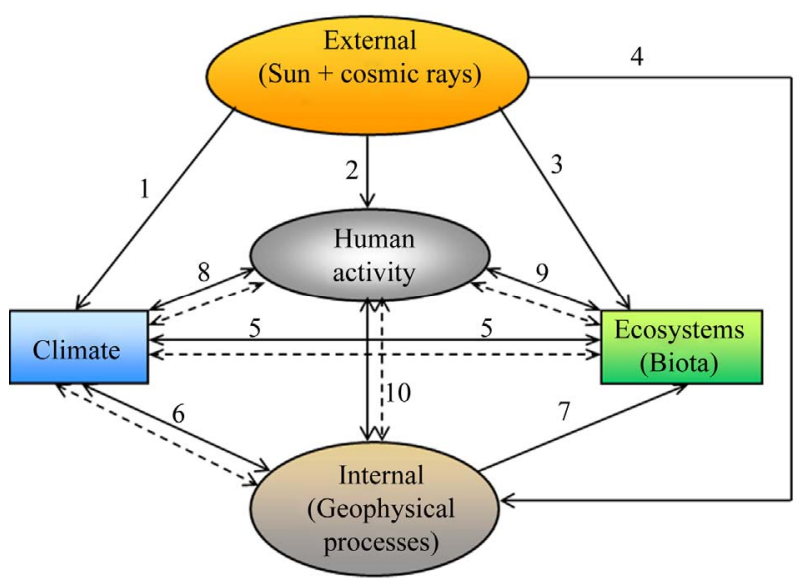

Figure 5. Generalized scheme of interactions in the Earth climate and ecosystems. 
tuations, which may be in different phase relation to forcing - from opposite till resonances in time and space. Third, there are many levels of impacts and mutual feedbacks, which can mix up in space and time: high-frequency impacts can lead to low-frequency consequences and vice versa. But the main difficulty is due to human activity.

Though it is generally accepted that human beings originated as a part of the Earth Nature, but the up-todate humankind can not exist in harmony with Nature because, for the sake of super excessive consumption, it breaks the loops of natural feedbacks at energetic, chemical and biological levels. Analysis of natural climateecosystems interactions shows that Nature foresaw many negative feedbacks to ensure limited fluctuations of climate and ecosystems parameters around some satisfactory mean values. In any case, we can understand and predict more or less accurately many natural processes on our planet. But it is almost impossible to understand, reasonably explain and predict the all spectrum of anthropogenic impacts, mainly because Man does not want to look honestly into his future looking only for maximum profit and comfort "here and now". That makes extremely difficult long-term prediction of the Earth System Dynamics and corresponding management with taking into account anthropogenic impacts.

Happily for scientists, we have an object in Nature without harmful feedbacks from human activity-the Sun. But who knows?

\section{Summary}

The actual state of the Earth climate is characterized by the World Ocean overheating and high land-ocean-atmosphere contrasts of heat content, leading to exceptionally high evaporation in oceans and inundations on contitnents, raised intensity of atmosphere circulation and extreme spatial-temporal dispersions of all climatic parameters.

Accumulating properties of the ocean strongly depend on the state of oceanic ecosystems. Due to ability of living matter to react to signals with extremely low energy (informational flows) mainly of solar origin, it can change physical properties of ocean waters and in this way control high-energetic macroscopic processes.

Considerable climate fluctuations can be due to "fossil" heat anomalies manifestation in upper layers with different lags after their origin by different mechanisms, solar activity impacts on oceanic biota among them.

Continuous evolution of living matter ensures nonstationarity of climate-ecosystem interactions, but contemporary quick trends in these systems hardly can be explained by internal or external forcing without taking into account human activity, which intrudes in Nature at ex- tremely high energetic, chemical and biological levels.

The Earth ecosystems, especially oceanic ecosystems, and human activity should be integrated into coupled ocean-atmosphere models to form the much more sophisticated general climate-ecosystems model for predicting the Earth future.

\section{REFERENCES}

[1] S. S. Lappo, S. K. Gulev and A. E. Rozdestvensky, "Large-Scale Heat Interaction in the Ocean-Atmosphere System and Energetically Active Zones of the World Ocean (Krupnomasshtabnoye Teplovoye Vzaimodeystvie v Systeme Okean-Atmosphera i Energoaktivniye Oblasti Mirovogo Okeana)," Gidrometeoizdat, Leningrad, 1990, 336 p.

[2] “Climate Change 2007: Synthesis Report," AR4 SYR. http://www.ipcc.ch/pdf/assessment-report/ar4/syr/ar4_syr _spm.pdf

[3] SIDC-Team, World Data Center for the Sunspot Index, Royal Observatory of Belgium, "Monthly Report on the International Sunspot Number," Online Catalogue of the Sunspot Index. http://www.sidc.be/sunspot-data/

[4] N. E. Huang, Z. Shen, S. R. Long, M. C. Wu, E. H. Shih, Q. Zheng, C. C. Tung and H. H. Liu, "The Empirical Mode Decomposition Method and the Hilbert Spectrum for Non-stationary Time Series Analysis," Proceedings of the Royal Society of London, Vol. 454, No. 1971, 1998, pp. 903-995. doi:10.1098/rspa.1998.0193

[5] J. Lean and D. Rind, "Climate Forcing by Changing Solar Radiation," Journal of Climate, Vol. 11, No. 12, 1998, pp. 3069-3094. doi:10.1175/1520-0442(1998)011<3069:CFBCSR >2.0.C $\underline{\mathrm{O} ; 2}$

[6] B. L. Diffey, "Solar Ultraviolet Radiation Effects on Biological Systems," Physics in Medicine \& Biology, Vol. 36, No. 3, 1991, pp. 299-328. doi:10.1088/0031-9155/36/3/001

[7] M. Vernet, "Symposium-in-Print: UV Effects on Aquatic and Coastal Ecosystems Introduction: Enhanced UV-B Radiation in Natural Ecosystems as an Added Perturbation Due to Ozone Depletion," Photochemistry and Photobiology, Vol. 82, No. 4, 2006, pp. 831-833.

doi:10.1562/2006-05-31-RA-907

[8] D.-P. Häder, "Does Enhanced Solar UV-B Radiation Affect Marine Primary Producers in Their Natural Habitats?" Photochemistry and Photobiology, Vol. 87, No. 2, 2011, pp. 263-266. doi:10.1111/j.1751-1097.2011.00888.x

[9] O. A. Troshichev and I. P. Gabis, "Variations of Solar UV Irradiance Related to Short-Term and Medium-Term Changes in Solar Activity," Journal of Geophysical Research, Vol. 103, No. A9, 1998, pp. 2659-2667.

[10] A. Gnanadesikan and W. G. Anderson. "Ocean Water Clarity and the Ocean General Circulation in a Coupled Climate Model," Journal of Physical Oceanography, Vol. 39, No. 2, 2009, pp. 314-332. doi:10.1175/2008JPO3935.1 
[11] G. Li, K. Gao and G. Gao, "Differential Impacts of Solar UV Radiation on Photosynthetic Carbon Fixation from the Coastal to Offshore Surface Waters in the South China Sea," Photochemistry and Photobiology, Vol. 87, No. 2, 2011, pp. 329-334. doi:10.1111/j.1751-1097.2010.00862.x

[12] F. Kaiser, "Coherent Oscillations in Biological Systems: Interaction with Extremely Low Frequency Fields," $R a-$ dio Science, Vol. 17, No. 5S, 1982, pp. 17S-22S. doi:10.1029/RS017i05Sp0017S

[13] X. F. Pang and B. Deng, "Investigation of Changes in Properties of Water under the Action of a Magnetic Field", Science in China Series G: Physics, Mechanics and Astronomy, Vol. 51, No. 11, 2008, pp. 1621-1632. doi:10.1007/s11433-008-0182-7

[14] R. Cai, H. Yang, J. He and W. Zhu, "The Effects of Magnetic Fields on Water Molecular Hydrogen Bonds," Journal of Molecular Structure, Vol. 938, No. 1-3, 2009, pp. 15-19.

[15] Y. M. Tourre and W. B. White, "ENSO Signals in Global Upper-Ocean Temperature," Journal of Physical Oceanography, Vol. 25, No. 6, 1995, pp. 1317-1332. doi:10.1175/1520-0485(1995)025<1317:ESIGUO > 2.0.C
$\underline{\mathrm{O} ; 2}$

[16] W. B. White, D. J. Cayan and J. Lean, "Global Upper Ocean Heat Storage Response to Radiative Forcing from Changing Solar Irradiance and Increasing Greenhouse Gas/Aerosol Concentration," Journal of Geophysical Research: Oceans, Vol. 103, No. C10, 1998, pp. 21355 21366.

[17] W. B. White and Y. M. Tourre, "A Delayed Action Oscillator Shared by the ENSO and QDO in the Indian Ocean," Journal of Physical Oceanography, Vol. 63, No. 2, 2007, pp. 223-241.

[18] W. B. White and Z. Liu, "Resonant Excitation of the Quasi-Decadal Oscillation by the 11-Year Signal in the Sun's Irradiance," Journal of Geophysical Research: Oceans, Vol. 113, No. C1, 2008, Article ID: C01002. doi:10.1029/2006JC004057

[19] L. Klyashtorin, "Pacific Salmon: Climate-Linked LongTerm Stock Fluctuations," PICES Press, Vol. 5, No. 2, 1997, pp. 30-34.

[20] J. J. Polovina, E. A. Howell and M. Abecassis, “Ocean's Least Productive Waters Are Expanding," Geophysical Research Letters, Vol. 35, No. 3, 2008, pp. 1-5. doi:10.1029/2007GL031745 